\title{
Did Randomized Clinical Trials (RCT) and Continually are Contributing Evidence for Progress in Radiation Oncology
}

\author{
Bogusław Maciejewski*, Leszek Miszczyk, Sławomir Blamek, Wojciech Majewski and Jerzy Wydmański \\ Department of Radiotherapy, Maria Skłodowska Curie Memorial Cancer Centre and Institute, Poland
}

Submission: January 23, 2018; Published: February 08, 2018

*Corresponding author: Bogusław Maciejewski, Department of Radiotherapy, Cancer Center-MSC Institute, Gliwice, 44-101 Gliwice, 15, Wybrzeze Armii Krajowej 15, Poland, Email: bmaciejewski@io.gliwice.pl

\begin{abstract}
Since clinical trials have been advocated as evidence based guidelines for radiotherapy many RCTs were carried out. Can they be considered compared with empirical studies, as a milestone progress in radiation oncology. Large heterogenous tumor sites and stages were enrolled into the trials on hyperoxygen therapy, radiosensitizers and altered fractionations raise some uncertainties and criticism regarding therapeutic gain usually reported as median end-points. Local tumor control rates have been and still are related to the tumor TNM status, but almost never to initial tumor volume (number of initial cancer stem cells) whereas the effect of irradiation is cell killing, not tumor stage killing. Many RCTs became disappointing or at least therapeutic gain has been lower than expected. Well known trials are reviewed and discussed. In contrary, some retrospective studies have provided important practical information's, i.g. tumor volume is more predictive to design dose fractionation than T stage, overall treatment time has been show as a strong determinant of treatment outcome. This finding initiated series of altered fractionation trials. Alpha/beta values for H\&N tumors and cell survival curves derived from skin cancer data were one of the first estimations based on empirical clinical studies. Identifying very low alpha/beta for prostate cancer has attracted stereotactic hypofractionated radiosurgery as an effective therapeutic modality. In this review we discuss the pros and cons of the trials and empirical studies and it looks they are complementary to one another.
\end{abstract}

Keywords: Randomized clinical trials; Empirical radiotherapy; Head and neck cancer

\section{Introduction}

In oncology, including radiotherapy, there is a general belief and paradigm that randomized clinical trials (RCTs) have been emerged as a major or even the only source of evidence based clinical guidelines. It means that any recommendations of specific medical procedures should rely on evidence for benefits and costs for patients. Different RCTs "evidences" have been uncritically for major changes in the treatment strategy. However, in physics cause-and-effect relationship is clear, whereas in oncology and radiotherapy is not simple and easy to be established. Genetic and biological nature of malignant tumors and patients who suffer from them is widely individual and complicated, and a final common pathway, if recognized, is initiated from wide range of possible triggers [1].

For over last 50 years' experience with RCTs in radiation oncology many uncertainties and doubts have been a rised, and important question, which expected to be is do they indeed play a major or rather modest role in the progress in clinical radiotherapy, and should they be continued based on classic protocols [2]. This topic was one of important discussion during conference of American Radium Society in May 2017.

\section{RCTs Uncertainties}

Well-known RCTs of head and neck tumors are reviewed to consider whether have they had an important impact on therapeutic gain in radiation oncology. Identifying hypoxic cells in human tumors various approaches were investigated to eliminate hypoxic cell subpopulation. Since middle 70 -ties to the middle of 80-ties to increase tumor oxygen delivery, and to strengthen efficacy of conventional dose fractionation, Hyperbaric Oxygen Therapy (HBO) was tested in 19 clinical trials (Table 1) which included all together about 2400 patients with various tumor sites and stages $[3,4]$. The early studies failed to show pronounced improvement in outcome, later studies gave conflicting results, with either showing some benefit or no significant gain. Cervix cancer was the only exception and the MRC trial showed $20 \%$ gain in the local tumor control in the HBO arm. However, it has been difficult to conclude whether this gain resulted from the HBO or due to the use of high fraction doses (Table 1). Because it was no longer easy to recruit a high number of patients to such trials, they were discontinued in favor of agents which specifically target the hypoxic cells and sensitize 
these cells to radiation. In vitro studies demonstrated that highly electron-affinic nitroaromatic compounds can preferentially radiosensitize hypoxic cells. Different compounds were tested, i.g. misomidasol, pimonidasol, sanazole and others. In this promising field, 18 trials which recruited about 5000 patients were designed and carried out. Once again, in the 15 trials no therapeutic gain was noted, except DAHANCA 5 trial in which

Table 1: Trial on physical or chemical radiation modifiers (LTC-Local Tumor Control).

\begin{tabular}{|c|c|c|c|}
\hline Trials & No. Trials & No.Pts & Results \\
\hline Hyperbaric Oxygen (HBO) & 19 & $\sim 2500$ & $\begin{array}{c}\text { No significant gain, except uterine } \\
\text { cervix (20\% } \uparrow \text { LTC) is it result of } \\
\text { HBO or high dose per fraction }\end{array}$ \\
\hline $\begin{array}{c}\text { Radio Sensitiers (nitroimidasoles) } \\
\text { Concurrent Chemo-Radiation } \\
\text { (H\&N) }\end{array}$ & 18 & $\sim 5400$ & $\begin{array}{c}15 \text { trials-no gain 7-15\% LTC } \\
\text { gain;DAHANCA 2 < 5 higher doses } \\
\text { of nitroimidasoles too neurotoxic }\end{array}$ \\
\hline
\end{tabular}

When overall treatment time (OTT) in radiotherapy for head and neck cancerswas recognized and well documented as one of a majordeterminant of therapeutic benefit [5-7], various altered fractionation schedules were designed as a challenge to conventional fractionation with relatively long OTTs. More than 33 RCTs, recruited over 11000 head and neck cancer patients, have been carried out for over 25 years. Putting it in a mildly way, expectation that one or some of altered schedules may become as a "Holy Grail" for head and neck cancers of various sites and stages seems to be a bit naive. If fact, some of the RCTs showed even inverse results $[8,9]$. CHART trial has shown average LTC gain of about $5 \%$ (which fell down to $0 \%$ after 10 -years of followup), and it was noted mainly for T3-T4 tumors, young patients ( $<50$ years) with well differentiated cancer, but not for T1-T2 cancers of oropharynx, oral cavity and hypopharynx. However, similar efficacy 54Gy in 12 days and 66Gy in 45 days, may suggest that "the shorter is as effective as the longer". Using L-Q nimorazole in head and neck cancer was used it resulted in 16\% improvement in the LTC [4]. Failure to note any benefit was generally caused by the fact that the drug doses were found too low, and a higher doses necessary for effective radiosensitization produced high risk of severe neurotoxicity. Once again, large series of these RCTs were more or less disappointing. model $\left(\alpha / \beta\right.$ value $=10 \mathrm{~Gy}$ ) the CHART NTD dose is $51.7_{\text {izoGy2.0 }}$ if given in 2.0Gy fractions. Total physical dose of 66Gy in control arm, corrected for accelerated repopulation (0.6Gy/day) above week 3 of irradiation [7], decreases to biologically equivalent dose (NTD) of $51.6_{\text {izoGyz. } .0}$. Therefore, biological equivalent doses were almost the same in both arms, and mature results of this trial surprisingly revealed a few percents higher gain in favor of the control arm [10]. On the contrary in the PMH trial 2.5 times higher LTC gain was noted for small tumors $(<4 \mathrm{~cm})$, and mainly for the hypopharynx (Table 2). Among many RCTs, the DAHANCA-7 trial is one of the most reliable since it included laryngeal cancersonly, and it was carried out only in the Danish cancer centers using the same protocol [11]. Due to shortening OTT by one week average $10 \%$ gain in the LTC was noted, but, for the select subgroup of well differentiated tumors the LTC increased to about $20 \%$.

Table 2: Selected randomized trials on altered dose fractionation for head and neck cancer regarding heterogeneity sites and $\mathrm{T} \& \mathrm{~N}$ stages (A-Accelerated, AH-Accelerated Hyperfractionation, $\mathrm{H}$ - Hyperfractionation).

\begin{tabular}{|c|c|}
\hline \multicolumn{1}{|c|}{ Trials } & Evidence And Uncertainties \\
\hline CHART (AH) & $\begin{array}{c}\text { Different T\&N and T localisations; 5\% LTC gain; after 10yrs-0\% mainly } \\
\text { for T3 T4, young pts, well differentiated tumors; no gain } \rightarrow \text { T1- T2, } \\
\text { orophx, oral cavity }\end{array}$ \\
\hline PMH (A) & Different T\&N and T sites \\
\hline MDACC 9003 (AH) & Low LTC gain; 2.5 x higher for T < 4cm and hypophx \\
\hline EORTC 22851 (AH) & Different T\&N and T sites moderate gain for Concomitant Boost \\
\hline EORTC 22791 (H) & Fairly homogeneous arms LTC gain moderate-for T3 not for T4 \\
\hline DAHANCA 7 (A) & $\begin{array}{c}\text { Single T site (larynx) LTC gain-10\%, higher for well differentiated } \\
\text { tumors }\end{array}$ \\
\hline
\end{tabular}

Evaluation of the RCTs results may lead to a bit confusing comparison (Table 3). In some trials the LTC rates in altered arm are close to the LTC in the control arm of other trials. Results of two meta analyses (MARCH 1 and 2) have clearly shown that previously awaited "Holy Grail" do not show-up [8,9]. Twenty one of the 50 trials (42\%) were potentially eligible, but finally, only 15 trials (30\%) with 6515 patients were included to the meta 
Table 3: Examples of altered arm results similar to control results in other trials.

\begin{tabular}{|c|c|c|c|}
\hline \multirow{2}{*}{ Study } & \multicolumn{2}{|c|}{ Local Tumor Control } & \multirow{2}{*}{ LTC Gain } \\
\cline { 2 - 3 } & Control Arm & Altered Arm & \\
\hline CHART & $40 \%$ & $45 \%$ & $5 \%$ \\
\hline RTOG 9003 & $46 \%$ & $54 \%$ & $8 \%$ \\
\hline DAHANCA 7 & $52 \%$ & $62 \%$ & $10 \%$ \\
\hline MDACC-CB & $66 \%$ & $79 \%$ & $13 \%$ \\
\hline EORTC 22791 & $40 \%$ & $59 \%$ & $19 \%$ \\
\hline
\end{tabular}

Important step forward in radiotherapy has been expected due to studies on molecular tumor profiles as predictors and prognostic factors. From Buffa et al. [12] analysis fairly homogenous CHART patient' clusters shown that configuration of negative p53, Bcl2-, with low Ki-67 and low CD31 predicts the LTC gain significantly higher than $5 \%$. It may suggest that therapeutic gain expected in many altered trials is hidden, and in fact it may exist not for all different $H \& N$ tumors but for clinically and biologically selected homogeneous group of patients.

Combined chemotherapy with radiation has raised a flurry of interest as attractive therapeutic modality regarding an improvement of both LTC and disease-free survival (DFS). Once again, meta analyses (MACH-NC, MACH-CH) showed $[13,14]$ lower than modest average therapeutic gain of only $4 \%$, (2\% improvement for neoadjuvant and adjuvant chemotherapy, and promising benefit of $12 \%$ for concurrent chemoradiation). Denis et al. [15] study on efficacy of chemoradiotherapy for advanced oropharyngeal cancer has shown 23\% increase in the 5-year LTC. This is a good example that when the RCT concentrates on single tumor type and site, therapeutic benefit can raise significantly. In contrary, when the RCTs include various tumor sites and stages which are the source of wide range of the initial number of cancer stem cells, then a real LTC gain can be washed away.

In a few trials misleading negative results have been reported. For example PORT meta analysis has shown no therapeutic benefit for postoperative radiotherapy for lung cancer patients. Thames (personal communication) reanalyzing the results of this meta analysis pointed out that a weak point was to mix together the $2 \mathrm{D}-{ }^{60} \mathrm{Co}$ and $3 \mathrm{D}$-conformal results. When his cut-off the ${ }^{60} \mathrm{Co}$ results and focused on the 3D trials only then reported negative results turned into significant $10 \%$ long-term therapeutic LTC gain.

Another example of the misleading results is the p-CAIR trial focused on accelerated (7-days-a week) versus conventional (5-days-a-week) postoperative radiotherapy for $\mathrm{H} \& \mathrm{~N}$ patients with high risk of local recurrence carried out by Suwinski et al. [16]. At the first glance, the authors noted no significant LRC difference between two arms of the trials. However, they designed own numerical molecular scoring system, i.e. high EGFR=1, low p53=1, low Ki-67=1, and low nm-23=1. For patients with total score higher than $2,40 \%$ increase in the LRC was found in favor of accelerated (7d/wk) schedule. Better outcome of HPV positive patients was well documented at least for oropharyngeal cancer, but this factor was not accounted for any previous trials.

\section{RCTs Statistics}

Generally, results of various fractionation schedules used in the trials do not allow to distinguish the effect of dose from that of the OTT. Survey of clinical papers in two journals (Radiother Oncol and J Radiat Oncol Biol Phys) has shown that only one-third of them contain a satisfactory description of how the dose was specified [17]. Although, authors generally agree that the RCTs in radiotherapy improve its efficacy, systemic interpretation of the results published in the literature is quite difficult and it should be taken with some grains of salt. Among 141 RCTs on therapy of advanced breast cancer (about 26,300 patients), reported on ASCO between 1984-1993, only three (2.1\%) showed significant benefit from the experimental treatment arm. It leads the readers to the impression that specific results recommended as evidence based, are in fact general but not specific guidelines, and how to treat individual patients is still considered as a statistically average persons.

There is no doubt that the LTC gain with increasing total dose is almost axiomatic for radiotherapy, but variations in natural tumor biology may often dominate over the effect of dose fractionation. In biostatistics of the RCTs the sample size is not only important, but also its nature also $[1,2,17,18]$. Methodological problems sometimes make conclusions uncertain and for that reason they are likely excluded from the meta analyses, (i.g. MARCH).

Factor as tumors biology, cell density, radiosensitivity, not constant rate of cell killing after daily fractions are highly heterogeneous. But, even if the RCTs are well designed and analyzed, the results could be somewhat puzzling since within a specific tumor stage TCP may differ significantly.

One of the Achilles heel of the RCTs seems to be the belief that relatively wide range of tumor sites and stages enrolled to each arm of the trial will alike respond to irradiation schedule. It's difficult to understand why TNM tumor stage still remains as axiomatic criterion of inclusion to the trial, whereas initial number of tumor stem cells (indirectly represented by tumor volume) is in fact radiation targets. Therefore it seems inconsistent to tailor dose fractionation to the $\mathrm{T}$ or $\mathrm{N}$ stage. This indisputable fact is however ignored. Although at the first glance, randomized arms may look homogeneous clinically but there is about 10 -fold difference in the initial number of tumor stem cells between the smallest and the largest tumors, within $\mathrm{T} 2$ stage, and much larger for higher $\mathrm{T}$ stages but a total dose given to all cases within each arm is the same. If, just by chance, in the control arm will be allocated more smaller tumors in each $\mathrm{T}$ category, and on the contrary, more larger tumors will be in the altered arm, it may likely lead to higher efficacy (higher LTC) in the control than in the altered one. Should such result be therefore considered as "an evidence" or rather as "an illusion"? Therefore it sounds logical that dose fractionation should be 
tailored to the volumetric staging which reflects number of the stem cells, but no longer to the $\mathrm{T}$ and $\mathrm{N}$ stages.

Usually the results of the trials are presented as an average and usually actuarial but not crude end-points in the selected time of the follow-up. Sometimes one can lose valuable information's that are spread around and beyond such point. Glatstein [1] called it as a "Tyranny of the median". Major problem with the median value is that the rest of survival curve is usually ignored. Furthermore, the probability for outcome is related to whole group but not for individuals. Nevertheless, Bentzen wittily and also rightly pointed out that "Evidence of lack of significance, does not necessarily means the lack of evidence". On the other hand, statistical significance not always corresponds with clinical importance.

Considering at least major doubts and uncertainties of the RCTs, there is not unanimous answer to the question whether randomized clinical trials should not be considered as evidence based milestones of progress in radiotherapy, although it may seem that they have played a modest role. Glatstein [1] has used elegant Latin proverb "Caveat Emptor", which means a kind of warning that one should be cautious to make unequivocal conclusions. Although evidence based guidelines are addressed to clinicians, evidence has to be measured and weighed carefully, and frequently, and it requires clinical experience, common sense and logic. It does not, however, means that evidence should be ignored and dismissed out from practical radiotherapy.

\section{Empirical Radiotherapy}

For about 10 decades empiricism has been a source of knowledge, growing experience and also progress in radiotherapy, but not as an alternative to RCTs. A few decades ago, Fowler mentioned that - "If radiotherapist had to await for fully scientific evidence basis for treating, for first patient radiotherapy would not have started yet". According to David Hume (XVIII century) "empircism means the best contact between one's understanding of knowledge and the world and it is not the point at which a mathematical proof crystalizes". In radiation oncology empiricism comes from generally accumulated clinical experience based, on "what has worked in the pastand what has not". Strong and important attribute of the empiricism are retrospective clinical studies, which for decades have been a source for growing clinical experience to select tumor volumes, dose and fractionation, timing, treatment techniques to design standards, long before the RCTs have developed.

In XX century, Gray Laboratory was a "Mecca" of experimental and clinical radiobiology. Almost all fundamental radiobiological mechanisms of tumor and normal tissue response to radiation were recognized, quantified as a basic rationale for clinical radiotherapy. In late 60 -ties Fletcher proposed modern clinical radiotherapy based on radiobiology principles which became a major milestone in radiotherapy. For example, utilizing own clinical observations, Fletcher pointed out that if dose per week is not higher than $10 \mathrm{~Gy}$, then in about $60 \%$ of $\mathrm{H} \& \mathrm{~N}$ cancer patients healing of the acute mucosal reaction (confluent mucositis) already occurs at the end of week 6 of conventional irradiation. His observations were confirmed by the results of many studies. In his time, accelerated repopulation was not discovered yet, but he intuitively and indirectly suggested that it plays important role in response of normal epithelium to fractionation, and the response is so intensive that effect of daily fraction of $2 \mathrm{~Gy}$ can be neutralized by this process.

During 60-ties and 70-ties two cancer centers in Poland gathered over 1000 skin cancer patients treated with one of the seven different fractionation schedules from a single dose of 18-25Gy to 70Gy in 47 fractions $[5,19]$. In none of the RCTs such wide range of doses has been used yet. In fact, it is methodolically not possible. This large set of non-randomized empirical retrospective data $[5,6,19,20]$, were later effectively used to:

a) Question reliability of the Strandquist formula for dose-time relationship and it showed that the exponent for time ( $\mathrm{T}$ ) in his formula should be higher than 0.33 . It was the first indirect sign that process of accelerated repopulation exist and it should not be ignored;

b) Demonstrate that tumor volume as a one of the major determinant of effective dose fractionation (Figure 1); (small tumors can be eradicated even by a single dose whereas larger needs a number of fraction doses);

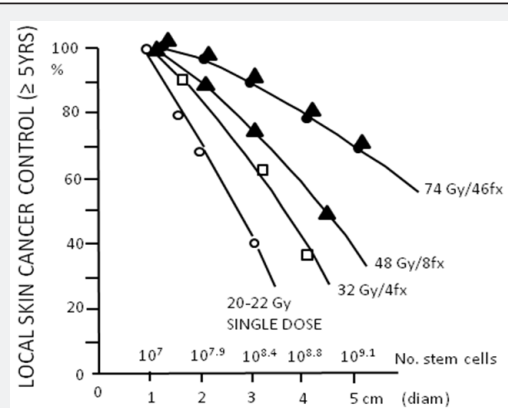

Figure 1: Impact of skin cancer size (initial number of cancer stem cells) and dose fractionation on local tumor control.

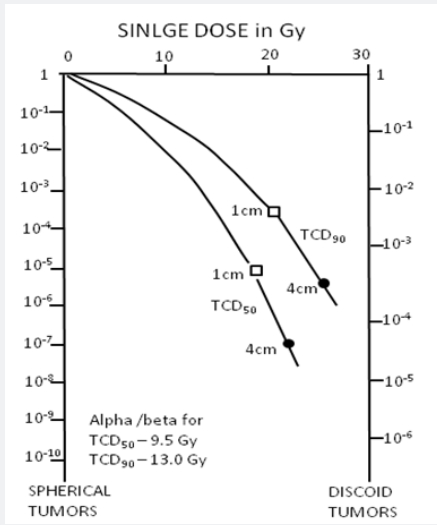

Figure 2: From "bedside to bench" - estimation of cell survival curve for skin cancer using retrospective data bank of about 1000 skin cancers (TCD50 - Tumor Cure Dose 50, TCD90 Tumor Cure Dose 90). 
c) To estimate cell survival curves fromclinical data as a bridge between bench and bed site (Figure 2);

d) Estimate alpha/beta values for skin cancer $(\alpha / \beta=$ 13.0Gy) and skin necrosis

( $\alpha / \beta=7.4 \mathrm{~Gy}$ ), which were one of the first published in the literature (Figure 3);

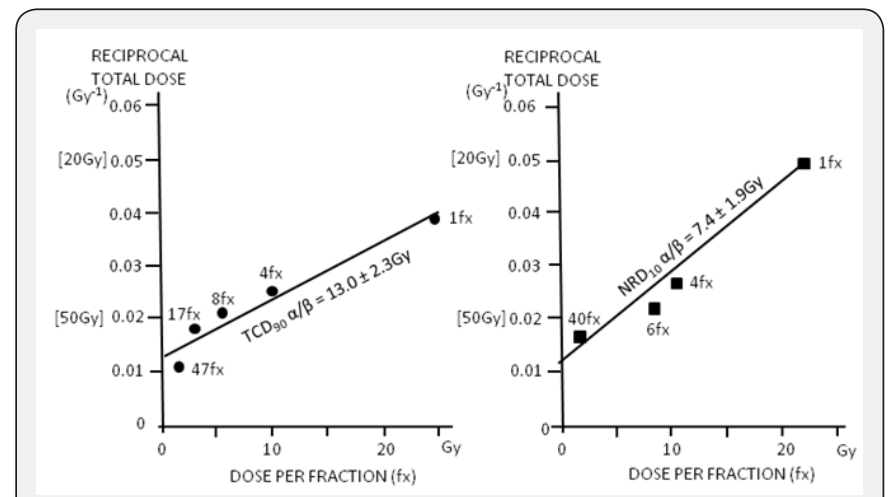

Figure 3: Local tumor control (3yrs)-Dose Intensity relationship for selected altered fractionation trials on head and neck cancer

e) Prove that time factor is much more important for treatment outcome than it was previously assumed and that exponent for time depends on OTT itself and in the Strandquist' and Ellis' formulas this factor was underestimated.

All these findings came from a single retrospective clinical data set.

Quantitative analyses of a large retrospective series of head and neck cancer irradiated in Gliwice have shown that overall treatment time (OTT) is one of the major determinant of treatment outcome. Cell kill effect caused by about 0.6Gy daily fraction of $2.0 \mathrm{~Gy}$ is neutralized by accelerated repopulation of the survived clonogenic cells $[6,7]$. Shortening the OTT results in an increase in the LTC, what has led to a milestone practical guideline that: "It is more effective to begin radiotherapy on Monday or Tuesday, and worse (never do it) to complete therapy on Monday or Tuesday" - (it means the last 2-3 fractions should be used as a second daily fraction in the previous week).

Although RCTs on altered fractionation failed to show pronounced therapeutic benefit, they were a source of important radiobiological information that accelerated repopulation previously estimated as a constant Drep $=0.6 \mathrm{~Gy} / \mathrm{day}$, in fact is increasing during irradiation and it depends on time OTT itself, with the increase to even 1.4-1.6Gy/days at the end of the week 6 of irradiation. It means that $10 \mathrm{~Gy}$ at the first week of irradiation is not biologically equivalent to $10 \mathrm{~Gy}$ at the week 6 , because in the week 6 cell kill effect of at least 3.5-4Gy (2.5 days x 1.4Gy) of $10 \mathrm{~Gy} /$ week is balanced by repopulation during weekend and thereafter [7]. The RCTs results have shown that attention should be focused on Dose Intensity (DI) (number of Gray per unit of time, e.g. number of Gy/day), which is more reliable parameter corresponding with treatment efficacy then Dose Escalation (DE). The latter one simply expresses an increase in physical dose. Although, 60Gy in 42 days, 70Gy in 49 days and 80Gy in 56 days illustrate dose escalation from 60Gy to 80Gy, but the same DI of these three schedules (DI $=1.43 \mathrm{~Gy} / \mathrm{d}$ ) means that they are biologically equivalent. Therefore, the DI seems more handy than DE to evaluate biological efficacy of different fractionation schedules (Figure 4). All these, practically useful information's came from empirical, often retrospective studies and have been used as rationale to design altered fractionation RCTs carried out through more than two decades.

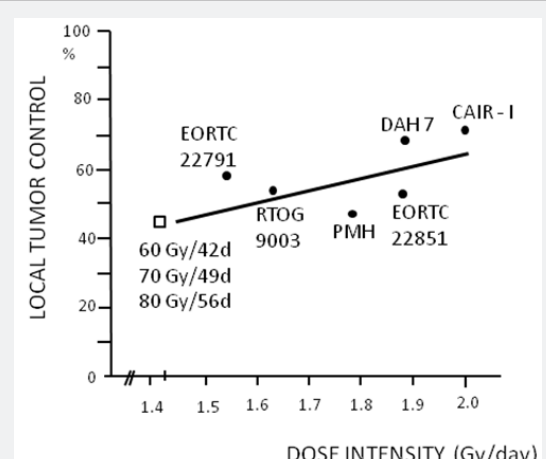

Figure 4: Impact of skin cancer size (initial number of cancer stem cells) and dose fractionation on local tumor control.

A few years ago, Fowler, has mentioned that radiotherapy is like a "Round Game", that means, some rules and methods abandoned in the past, nowadays are coming back to the market. Fowler together with Ritter \& Bentzen [21-23] using retrospective data have estimated unexpectedly low alpha/beta value of less than 2.0Gy for prostate cancer.

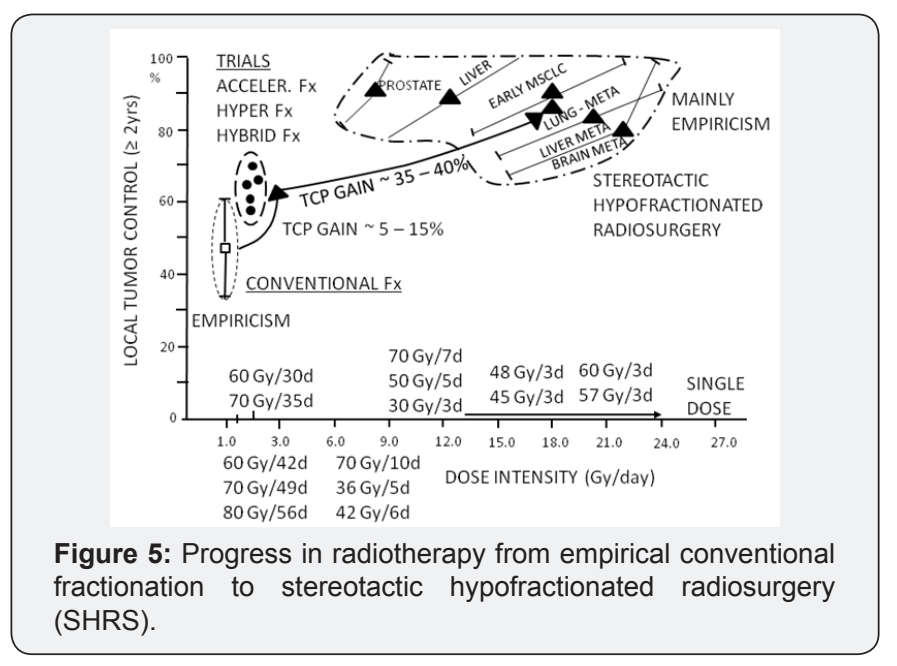

Revival a single dose or few large fractions as a high-tech stereotactic radiotherapy has been a real milestone "back to future" in radiotherapy. Nowadays, large single dose of 8-29Gy or a few fractions of 7 up to even 20Gy are more and more widely used as radical therapy of primary or metastatic brain tumors, head and neck, lung, pancreas, liver or prostate cancer. Results look spectacular and provide high rate of $80-95 \%$ of at least 2 year LTC. This is undoubtedly an important milestone in 
radiotherapy, with its empirical roots. Figure 5 illustrates this enormous "gain-jump" in curative radiation oncology, although a majority studies have a long empirical history and those on stereotactic hypofractionated radiotherapy also remains mainly empirical and RCTs just have begun. Despite many years of empirical and randomized studies, somewhat epical question "Mirror, mirror at the wall-tell which therapy is the best at all" -still remains unanswered.

\section{Conclusion}

During many decades of radiotherapy we have learned that "cause-and-effect relationships" are not simple. Malignant tumors and humans biology is widely individual, and recognizable final common pathway is initiated from wide range of possible triggers [1]. Nobody can settle that whether empirical radiotherapy is advantageous or inferior alternative to the RCTs, because in fact they are complementary to one another. Empirical retrospective studies should not be ignored as a source of practical importance although they are not randomized.

The RCTs results, even significant, are not always useful to design individually personalized radiotherapy alone or as a part of systemic therapy. It should be remembered that about $80 \%$ of patients stay out of any RCTs. There is plausible expectation that genomics, proteomics and molecular tumor profiles shell influence philosophy and tailoring radiation oncology. Despite of preliminarily unsatisfied results of the CHART or p-CAIR show that molecular profiles allowed to select well defined clusters of patients with the LTC much higher than average median. Already translation research strongly influences present and prospective progress in radiotherapy. There is no doubt the RCTs should be continued to check and prove empirical findings but its methodological rules and criteria need likely to be updated. It seems that so-called "Feedback Trial" might be a reasonable solution. It means, that patients with molecular profiles estimated prior to therapy should be enrolled into the trial and randomized based on volumetric, but not TNM staging. Longterm results categorized as winners (cured) or losers (failures) should be confronted back with molecular profiles within each category and cross-checked for winners and losers to define specific molecular and/or genetic markers being a strong specific predictors for each end-point. It likely seems the only rational way to move from averages to evidence bases, individually personalized effective radiation therapy.

\section{References}

1. Glatstein E (2004) Personal thoughts on statistics, lies, damn lies and (oncologic) statistics. Int J Radiat Oncol Biol Phys 58(5): 1329-1333.

2. Bentzen SM (1998) Towards evidence based radiation oncology: improving design, analysis, and reporting clinical outcome studies in radiotherapy. Radiother Oncol 46(1): 5-18.

3. Overgaard J, Horseman MR (1996) Modification of hypoxic induced radioresistance in tumors by the use of oxygen and sensitizers. Sem Radiat Oncol 6(1): 10-21.

4. Horsman MR, Overgaard J (2016) The impact of hypoxia and its modification of the outcome of radiotherapy. J Radiat Res 57 Suppl 1: i90-i98.

5. Trott KR, Maciejewski B, Preuss-Bayer G, Skołyszewski J (1984) Dose response curve and split-dose recovery in human skin cancer. Radiother Oncol 2(2): 123-127.

6. Hliniak A, Maciejewski B, Trott KR (1983) The influence of the number of fractions, overall treatment time and field size on the local control of Cancer of the skin. British J Radiat 56(668): 569-598.

7. Withers HR, Taylor JMG, Maciejewski B (1988) The hazard of accelerated tumor clonogen repopulation during radiotherapy. Acta Oncol 27(2): 181-191.

8. Bourhis J, Overgaard J, Audry H, Ang KK, Saunders M, et al. (2006) Hyperfractionated or accelerated radiotherapy in head and neck cancer: a metaanalysis. Lancet 368(9538): 843-854.

9. Lacas B, Bourhis J, Overgaard J, Zhang Q, Gregoire V et al. (2017) Role of radiotherapy fractionation in head and neck cancers (MARCH): an updated meta-analysis. Lancet Oncol 18(9): 1221-1238.

10. Saunders MI, Rojas AM, Parmar MK, Diche S (2010) Mature results of a randomized trial of accelerated hyperfractionated versus conventional radiotherapy in head and neck cancer. Int J Radiat Oncol Biol Phys 77(1): 3-8.

11. Overgaard J, Hansen HS, Specht L, Overgaard M, Grau C et al (2003) Fie compared with six fractions per week of conventional radiotherapy of squamous cell carcinoma of head and neck: DAHANCA 6\&7 randomized controlled trial. Lancet 362(9388): 933-940.

12. Buffa F, Bentzen SM, Daley F, Dische S, Saunders MI, et al. (2004) Molecular marker profiles predict locoregional control of head and neck squamous cell carcinoma in a randomized trial Continuous hyperfractionated accelerated radiotherapy. Clin Cancer Res 10(11): 3745-3754.

13. Pignon JP, Bourhis J, Domenge C, Designé L (2000) Chemotherapy added to locoregional treatment for head and neck squamous cell carcinoma. Three meta-analysis of updated individual data. MACH-NC Collaborative Group. Meta-Analysis of chemotherapy on head and neck cancer. Lancet 355(9208): 949-955.

14. Blanchard P, Baujat B, Holostenco V, Bourredjem A, Baey C, et al. (2011) Meta-analysis of chemotherapy in head and neck cancer (MACH-CH): a comprehensive analysis by tumor site. Radiother Oncol 100(1): 33-40.

15. Denis F, Garaud P, Bardet E, Alfonsi M, Sire C, et al. (2004) Final results of the 94-01 French Head and Neck Oncology and Radiotherapy Group randomized trial comparing radiotherapy alone with concomitant radiochemotherapy in advanced stage oropharynx carcinoma. J Clin Oncol 22(1): 69-76.

16. Suwinski R, Jaworska M, Nikiel B, Woźniak G (2011) Postoperative continuous 7-days-a-week radiotherapy for high-risk squamous cell cancer of the head and neck. Long-term results of a randomised clinical trial. Int J Radiat Oncol Biol Phys 81: 104-110.

17. Marsh MJ, Hawkins BS (1994) Publications from multicenter clinical trials: statistical techniques and the accessibility to the reader. Stat Med 13(23-24): 2393-2406.

18. Bentzen SM (1994) Radiobiological considerations in the design of clinical trials. Radiother Oncol 32(1): 1-11.

19. Maciejewski B, Taylor JMG, Withers HR (1986) Alpha/beta value and the importance of size of dose per fraction for late complications in the supraglottic larynx. Radiother Oncol 7(4): 323-326.

20. Maciejewski B, Skates S, Zajusz A, Lange D (1993) Importance of tumor size and repopulation for radiocurability of skin Cancer. Neoplasma 40(1): 51-54.

21. Fowler JF, Chappell R, Ritter MA (2001) Is alpha/beta for prostate tumors really low? Int J Radiat Oncol Biol Phys 50(4): 1021-1031. 
22. Fowler JF, Ritter MA (1995) A rationale for fractionation for slowly proliferating tumors such as prostatic adenocarcinoma. Int J Radiat Oncol Biol Phys 32(2): 521-529.
23. Bentzen SM, Ritter MA (2005) The alpha/beta ratio for prostate cancer: what is it, really? Radiother Oncol 76(1): 1-3.

\section{Your next submission with Juniper Publishers will reach you the below assets}

- Quality Editorial service

- Swift Peer Review

- Reprints availability

- E-prints Service

- Manuscript Podcast for convenient understanding

- Global attainment for your research

- Manuscript accessibility in different formats

( Pdf, E-pub, Full Text, Audio)

- Unceasing customer service

Track the below URL for one-step submission https://juniperpublishers.com/online-submission.php 